\title{
Fusion: a true challenge for an enormous reward
}

\author{
J. ONGENA
}

Plasma Physics Laboratory, Royal Military Academy

Renaissancelaan 30, 1000 Brussels, Belgium

\begin{abstract}
Summary. - Nuclear physics shows that energy can be released from both fission of heavy nuclei and fusion of light nuclei. Steady progress shows that fusion an important additional option for energy production in the future - promises to be a clean and safe solution for mankind's long-term energy needs with minimal environmental impact. A source of energy which would be inexhaustible, inherently safe and environmentally friendly, is this not a marvellous prospect? Nuclear fusion, a possible candidate for this role, has been the energy source of our Sun and the stars in the universe for billions of years. This process requires temperatures of tens of millions of degrees, so extremely high and foreign to our daily experience that it seems out of reach. Nevertheless, these extremely high temperatures are routinely realised in several laboratories all over the world, and since the early 1990s, tens of MW fusion power have been released from fusion reactions. We are witnessing the birth of a new technology destined to meet the gigantic future energy needs of mankind with minimal impact on the environment.
\end{abstract}

\section{1. - Fusion reactions in our Sun and on Earth}

The dominant fusion reaction in our Sun converting hydrogen into helium is the p-p fusion chain, the other being the carbon-nitrogen-oxygen or CNO cycle. The CNO cycle is dominant in stars that are about 1.3 times heavier than our Sun; only about $1.7 \%$ of the ${ }^{4} \mathrm{He}$ nuclei in our Sun are estimated to originate from the CNO cycle. 
At first glance it seems easy to overcome the repulsion of the positively charged nuclei involved in fusion reactions. Why not simply use a particle accelerator to provide the nuclei with sufficient energy and make them collide? Unfortunately, the probability for a fusion reaction is extremely small. Rather than fuse, the nuclei are mostly scattered in all directions and only few fusion reactions take place.

Our Sun offers a more promising solution: its whole mass of gas is at a high temperature enabling a much larger number of fusion reactions to occur per unit of time. Gravity is responsible for confinement and heating of the fusing protons. An estimate of the temperature in the centre of the Sun can be found from the observation that the protons need to be sufficiently fast (i.e. have sufficient energy and thus be sufficiently hot) to overcome the compressional forces from gravitation. This means that kinetic energy of a proton in the centre = potential energy from gravity, or

$$
\begin{aligned}
\frac{3}{2} k T_{\text {proton in centre }} & =\frac{G m_{\text {proton }} M_{\text {Sun }}}{R_{\text {Sun }}}, \\
T_{\text {proton in centre }} & =\frac{2 G m_{\text {proton }} M_{\text {Sun }}}{3 k R_{\text {Sun }}},
\end{aligned}
$$

with $k=1.38 \times 10^{-23} \mathrm{~J} / \mathrm{K}$ Boltzmann's constant, $G=6.67 \times 10^{-11} \mathrm{Nm}^{2} / \mathrm{kg}^{2}$ the gravitational constant, $M_{\text {Sun }}=1.989 \times 10^{30} \mathrm{~kg}$ and $R_{\text {Sun }}=695500 \mathrm{~km}=6.955 \times 10^{8} \mathrm{~m}($ more than 2 times the distance Earth-Moon ...) we obtain

$$
T_{\text {protons in centre }}=1.56 \times 10^{7} \mathrm{~K}=15.6 \mathrm{MK} .
$$

Fusion of heavier nuclei in stars will occur at higher temperatures. From the formula above, it is immediately clear that a contraction of the star under its own gravity will further raise the central temperature. Fusion in heavy stars will continue to keep the star alive as long as the reactions are exothermic and thus are able to help the star defend itself against a full collapse under its own gravity. The mass defect curve (fig. 1) shows that energy can be gained from fusion reactions up to ${ }^{62} \mathrm{Ni}$, which is the tightest bound nucleus [1]. Once the star tries to fuse heavier nuclei, gravity finally wins, and a collapse of the star follows. This ends in a spectacular supernova, leading to a neutron star or for the heaviest stars, the formation of a black hole.

The p-p and CNO fusion reactions in our Sun have a much too low reaction rate to be useful on Earth. One of the underlying reasons is that for the formation of a ${ }^{4} \mathrm{He}$ nucleus two protons have to be converted into two neutrons, involving a slow beta-plus decay reaction. Despite this fact, every seconds about 700 million tonnes of $\mathrm{H}$ are converted in our Sun into 695 million tonnes of ${ }^{4} \mathrm{He}$; it thus means that every second about 5 million tonnes of the Sun's mass disappear in the form of radiation [1]!

Thus, on Earth we have to use nuclear fusion reactions that avoid the conversion from protons to neutrons, i.e. use as reaction products light atomic nuclei that already contain neutrons, needed for the formation of ${ }^{4} \mathrm{He}$. Thus, as a substitute for the p-p fusion cycle in the Sun, we need to make use of the isotopes D and T or possibly also 


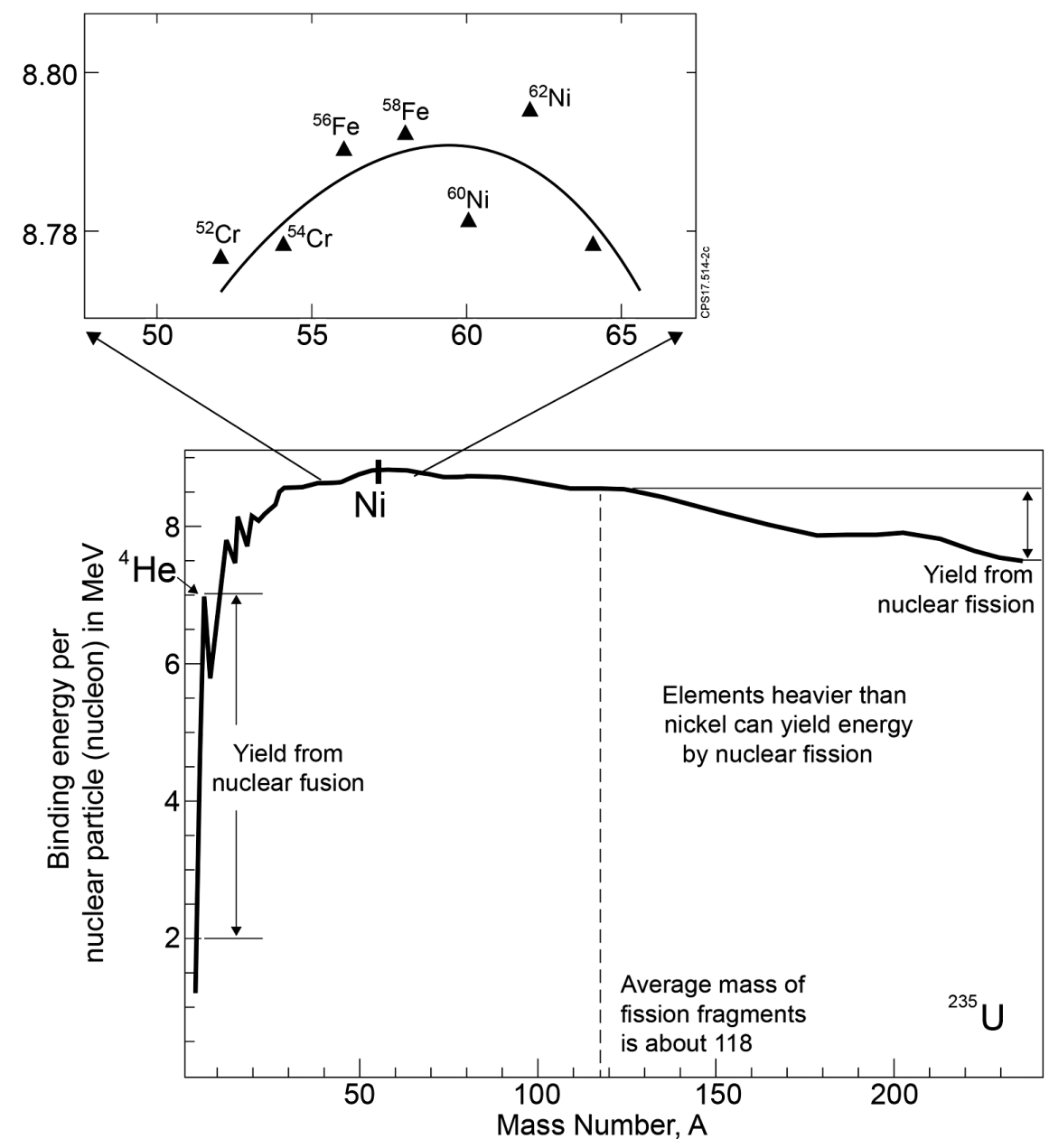

Fig. 1. - Binding energy per nucleon versus atomic mass number showing the maximum in the curve reached for ${ }^{62} \mathrm{Ni}$. Note the exceptionally large binding energy of the ${ }^{4} \mathrm{He}$ nucleus among the light nuclei. An approximate indication is given for the difference in binding energy per nucleon in fusion reactions of light nuclides with ${ }^{4} \mathrm{He}$ as one of the reaction products $(\sim 5 \mathrm{MeV} /$ nucleon$)$ and fission of ${ }^{235} \mathrm{U}(\sim 0.9 \mathrm{MeV} /$ nucleon$)$. The exact numbers for the mass defects of selected nuclides with mass numbers up to 20 can be found in the appendix.

the rare isotope ${ }^{3}$ He. Here D symbolizes deuterium (the stable isotope of hydrogen with a nucleus consisting of one proton and one neutron) and $\mathrm{T}$ is the symbol for tritium (the radioactive hydrogen isotope with a nucleus of 2 neutrons and 1 proton). A number of fusion reactions [2] together with their cross-sections and energy released are listed in table I. Note that the energy released is the largest in reactions where a ${ }^{4} \mathrm{He}$ nucleus is formed because of its exceptionally large binding energy (fig. 1). The fusion reaction 
TABLE I. - Fusion reactions.

\begin{tabular}{|c|c|c|c|c|}
\hline Reaction & $\begin{array}{c}\sigma \text { at } 10 \mathrm{keV} \\
\text { (barn) }\end{array}$ & $\sigma_{\max }$ (barn) & $\begin{array}{c}\text { Center-of-mass } \\
\text { energy }(\mathrm{keV}) \text { for } \\
\sigma_{\max }\end{array}$ & $\begin{array}{c}\text { Energy released } \\
(\mathrm{MeV})\end{array}$ \\
\hline $\mathrm{D}+\mathrm{T} \rightarrow{ }^{4} \mathrm{He}+\mathrm{n}$ & $2.72 \times 10^{-2}$ & 5.0 & 64 & 17.59 \\
\hline $\mathrm{D}+\mathrm{D} \rightarrow \mathrm{T}+\mathrm{p}$ & $2.81 \times 10^{-4}$ & 0.096 & 1250 & 4.04 \\
\hline $\mathrm{D}+\mathrm{D} \rightarrow{ }^{3} \mathrm{He}+\mathrm{n}$ & $2.78 \times 10^{-4}$ & 0.11 & 1750 & 3.27 \\
\hline $\mathrm{T}+\mathrm{T} \rightarrow{ }^{4} \mathrm{He}+2 \mathrm{n}$ & $7.90 \times 10^{-4}$ & 0.16 & 1000 & 11.33 \\
\hline $\mathrm{D}+{ }^{3} \mathrm{He} \rightarrow{ }^{4} \mathrm{He}+\mathrm{p}$ & $2.2 \times 10^{-7}$ & 0.9 & 250 & 18.35 \\
\hline $\mathrm{p}+{ }^{6} \mathrm{Li} \rightarrow{ }^{4} \mathrm{He}+{ }^{3} \mathrm{He}$ & $6 \times 10^{-10}$ & 0.22 & 1500 & 4.02 \\
\hline $\mathrm{p}+{ }^{11} \mathrm{~B} \rightarrow 3{ }^{4} \mathrm{He}$ & $4.6 \times 10^{-17}$ & 1.2 & 550 & 8.68 \\
\hline $\mathrm{p}+\mathrm{p} \rightarrow \mathrm{D}+\mathrm{e}^{+}+\nu$ & $3.6 \times 10^{-26}$ & - & - & $1.44+0.27(\nu)$ \\
\hline $\mathrm{p}+{ }^{12} \mathrm{C} \rightarrow{ }^{13} \mathrm{~N}+\gamma$ & $1.9 \times 10^{-26}$ & $1.0 \times 10^{-4}$ & 400 & 1.94 \\
\hline
\end{tabular}

that results in the formation of ${ }^{4} \mathrm{He}$ and has the largest cross-section is the so-called D-T reaction:

$$
\mathrm{D}+\mathrm{T} \rightarrow{ }^{4} \mathrm{He}(3.5 \mathrm{MeV})+\mathrm{n}(14.1 \mathrm{MeV})
$$

In this fusion reaction, "only" a rearrangement of the nuclides is needed to form ${ }^{4} \mathrm{He}$, which is much easier to realize: the cross-section $\sigma$ for this reaction at e.g. $10 \mathrm{keV}$ is about $10^{24}$ times larger than that for the p-p reaction.

The reaction products are an $\alpha$-particle ( ${ }^{4}$ He nucleus) and a neutron. Twenty percent of the reaction energy is carried by the $\alpha$-particles and $80 \%$ by the much lighter neutron. In magnetic confinement systems the neutron does not feel the presence of the magnetic field (because it is not charged) and escapes immediately from the reactor volume, while the charged $\alpha$-particle remains confined by the magnetic field. The kinetic energy of these escaping fast neutrons will be converted into heat in a blanket and then into electricity using conventional technology (steam cycle). About one million times more energy is released from a fusion reaction in comparison with a chemical one (MeV's instead of eV's for the latter). This is the reason why so little fuel can produce so much energy: when burnt in a fusion reactor, the deuterium contained in 1 litre of water (about $33 \mathrm{mg}$ ) will produce as much energy as burning 260 litres of gasoline.

Other possible fusion reactions of interest between isotopes of hydrogen and helium are

$$
\begin{aligned}
& \mathrm{D}+\mathrm{D} \rightarrow{ }^{3} \mathrm{He}(0.82 \mathrm{MeV})+\mathrm{n}(2.45 \mathrm{MeV}) \\
& \mathrm{D}+\mathrm{D} \rightarrow \mathrm{T}(1.01 \mathrm{MeV})+\mathrm{H}(3.02 \mathrm{MeV}) \\
& \mathrm{D}+{ }^{3} \mathrm{He} \rightarrow{ }^{4} \mathrm{He}(3.6 \mathrm{MeV})+\mathrm{H}(14.7 \mathrm{MeV})
\end{aligned}
$$




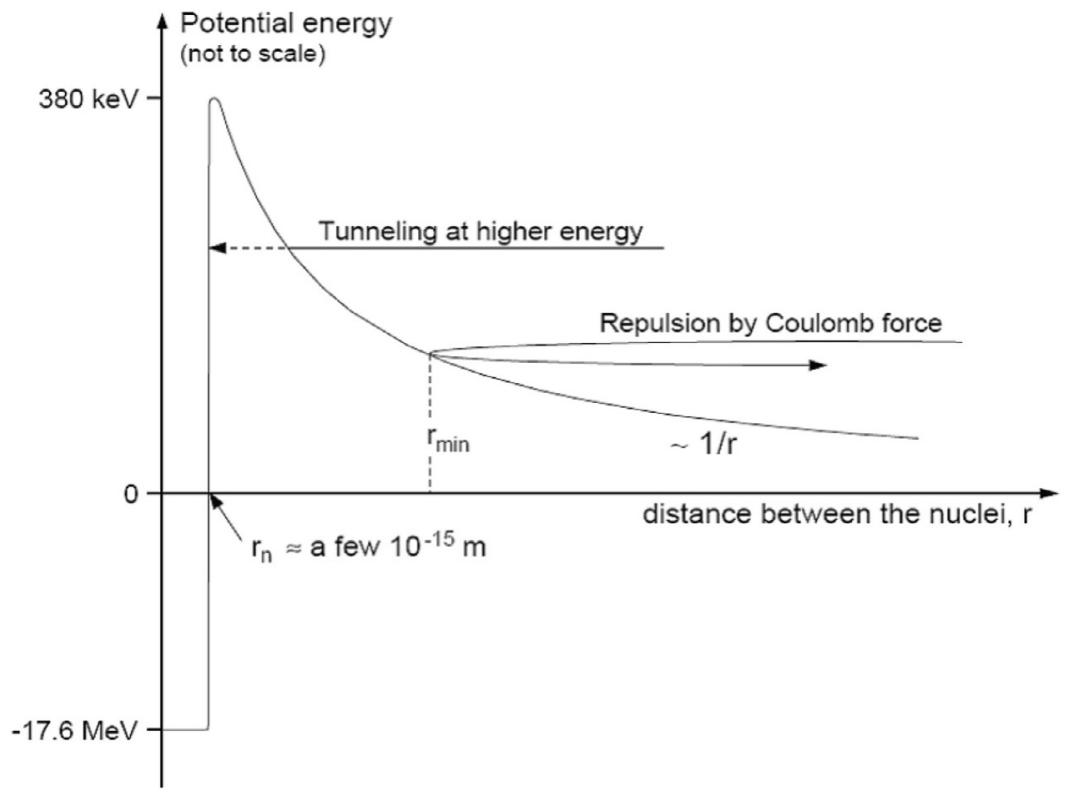

Fig. 2. - Atomic particles can overcome the Coulomb barrier (electrostatic repulsion) at much lower energies than the maximum by "tunnelling" through it. This increases the probability for fusion.

These are more difficult to achieve and have a lower power density than the D-T reaction $[3,4]$ but show even more benign environmental features. The D-D reaction would eliminate the need for tritium and produce neutrons with lower energies which are therefore easier to absorb and shield. A reactor based on the $\mathrm{D}-{ }^{3} \mathrm{He}$ reaction would proceed with low neutron production (some neutrons would be produced in competing but much less occurring D-D reactions) and lead to much less induced radioactivity in the reactor structures. However, the prospects for these "advanced" fuels are still too speculative and only the D-T reaction has immediate future prospects. For the interested reader, the appendix provides the masses and binding energies per nucleon for a selection of lighter nuclides, including those involved in reactions (4) to (7).

In order to undergo fusion reactions, the reacting particles have to approach each other to within short distances. A first estimate of the energy necessary to realise fusion reactions can be obtained from the potential energy of one nucleus in the presence of the electric field of another one at a distance such that, in the classical representation, the nuclei just touch each other. The maximum of the Coulomb barrier between two nuclei with radius $R_{1}$ and $R_{2}$ and charges $q Z_{1}$ and $q Z_{2}$ can be found from

$$
V_{C}[\mathrm{~J}]=\frac{q^{2} Z_{1} Z_{2}}{4 \pi \varepsilon_{0}\left(R_{1}+R_{2}\right)},
$$




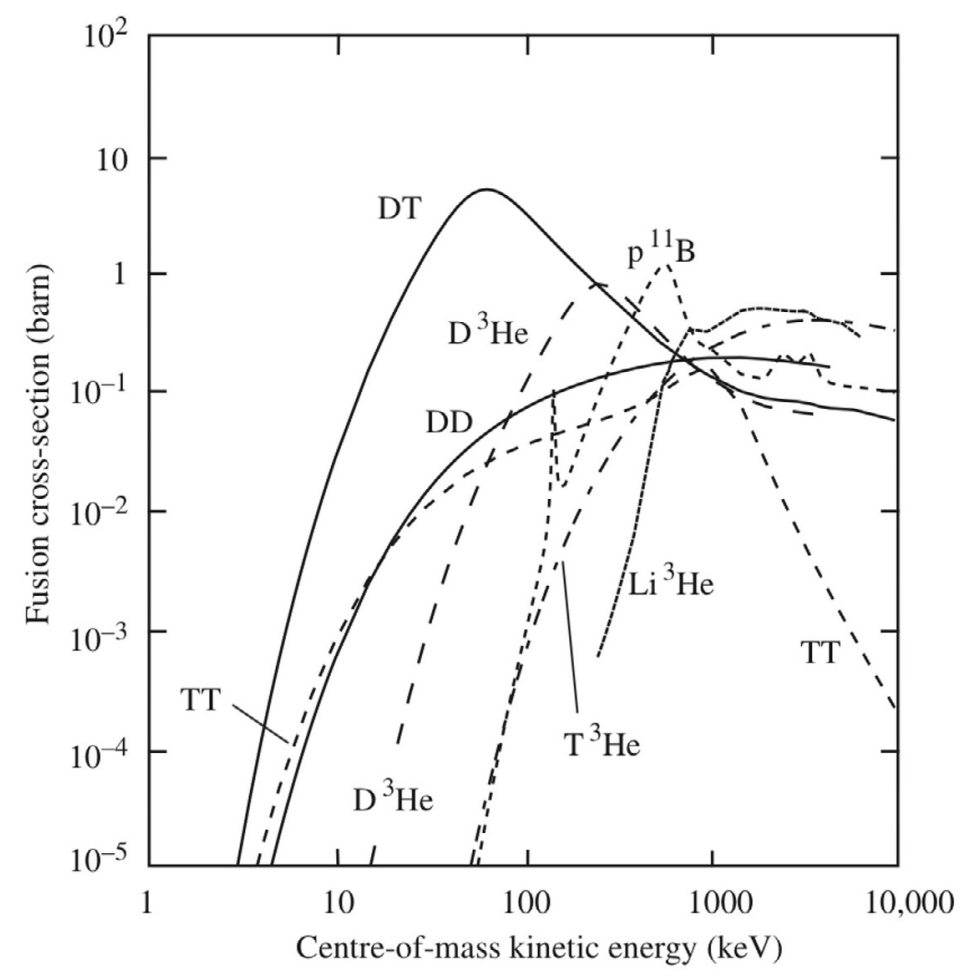

Fig. 3. - Cross-sections (in barn) as a function of the centre-of-mass energies of the reacting particles for various fusion reactions.

or

$$
V_{C}[\mathrm{MeV}]=1.44 \frac{Z_{1} Z_{2}}{1.4\left(A_{1}^{1 / 3}+A_{2}^{1 / 3}\right)},
$$

where we made use of the formula for the radius $R$ of the nuclei [5]:

$$
R=1.4 A^{1 / 3} \times 10^{-15} \mathrm{~m}
$$

For the reaction between deuterium and tritium we find $0.38 \mathrm{MeV}$. In the classical picture, nuclei should therefore at least acquire this energy before any fusion reaction can take place. The equivalent temperature of a plasma gas with this value as average energy amounts to more than 4 billion degrees.

A reduction of this huge temperature is made possible by the tunnel effect. Owing to the wave character of matter, nuclei have a finite probability to "tunnel" through the Coulomb barrier and thus can fuse at energies much lower than those given by the maximum of the barrier (fig. 2). As we shall see further, this reduction is substantial, and the optimum temperature for a magnetically confined D-T plasma is about $13 \mathrm{keV}$. 


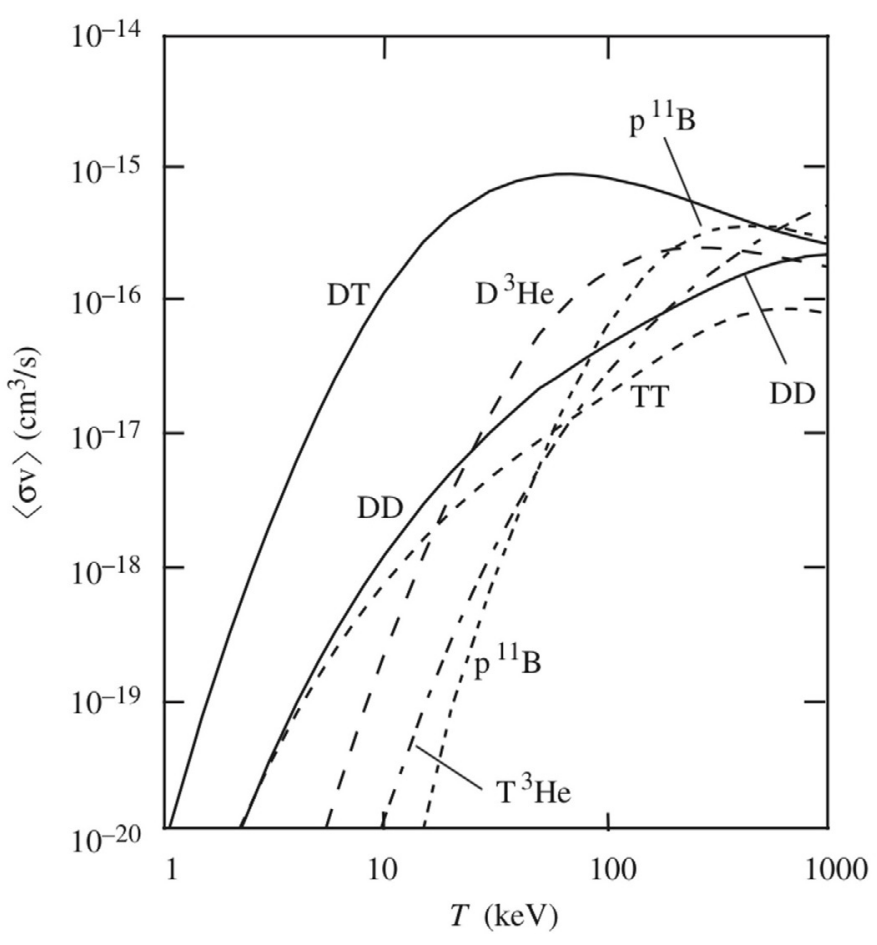

Fig. 4. - Reactivity averaged over a Maxwell-Boltzmann distribution as a function of temperature for the fusion reactions of fig. 3 .

Cross-sections [2] as a function of the centre-of-mass energies of the reacting particles for the reactions (4) to (7) above are shown in fig. 3. The largest cross-section is found for the D-T reaction (about 5 barn) at the centre-of-mass energy of the colliding particles $1 / 2 \mu\left(v_{1}-v_{2}\right)^{2}$ (where $\mu=1 / 2\left(m_{1} m_{2} /\left(m_{1}+m_{2}\right)\right.$ ) of about $60 \mathrm{keV}$. However in a gas one has to take into account that the velocities of the colliding particles are described by a Maxwell-Boltzmann distribution and values for the reactivity $\langle\sigma v\rangle$ averaged over this distribution as a function of the temperature are shown in fig. 4 for various fusion reactions. The maximum is now much broader but still around energies of about $60 \mathrm{keV}$ for the D-T reaction. Thus one is inclined to conclude that the plasma has to be heated to temperatures of over 600 million degrees to obtain the maximum fusion rate. However, one has to recall that in a magnetic fusion device, tokamak or stellarator, the operating pressure $p=n k T$ is constant, and that therefore there is an inverse relation between temperature and density. The fusion reaction rate $R=\frac{n^{2}}{4}\langle\sigma v\rangle$ can thus also be written as $R=\frac{1}{4} \frac{\langle\sigma v\rangle}{T^{2}}$ and the operating temperature of a reactor will be determined by the maximum of this function [6] as shown in fig. 5. It is clear that for the D-T reaction the peak is located at about $13 \mathrm{keV}$. For the $\mathrm{D}-\mathrm{D}$ or $\mathrm{D}^{3}{ }^{3} \mathrm{He}$ reactions, the temperature is higher or the power density is smaller at the maximum of the $\langle\sigma v\rangle$ curve. For the D-T reaction 


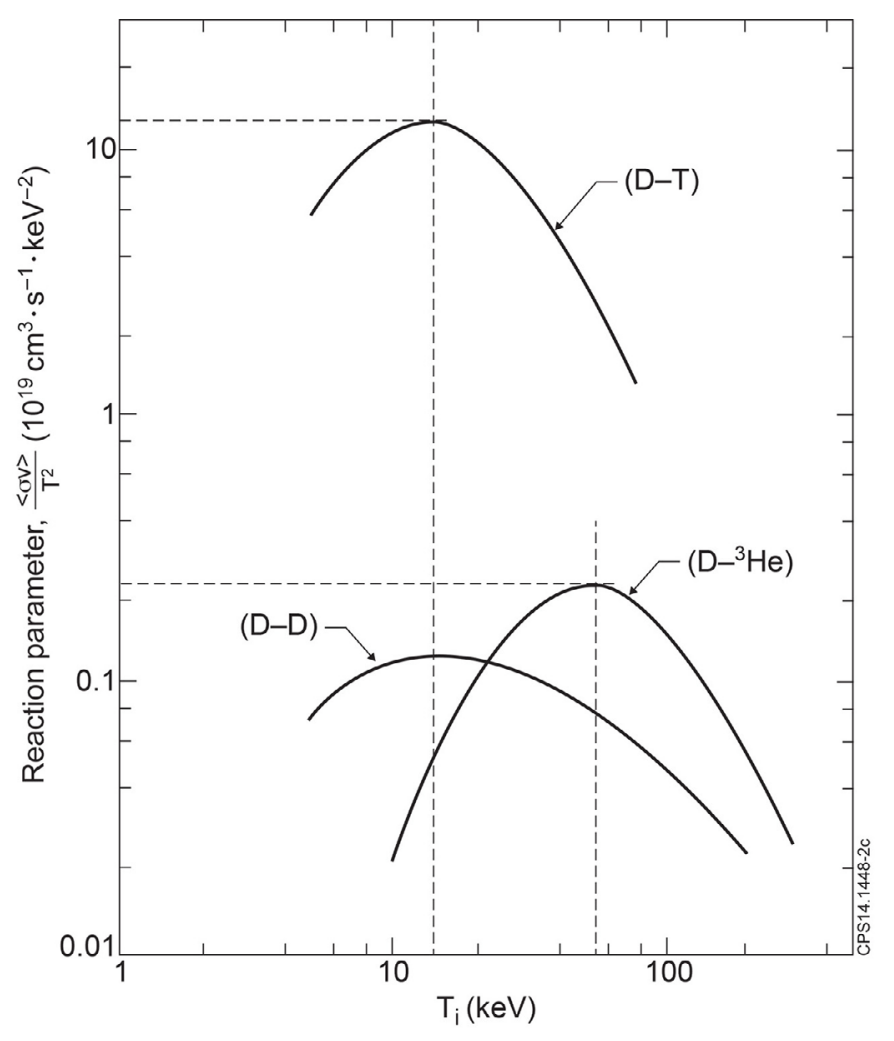

Fig. 5. - Maxwellian averaged values for $\frac{\langle\sigma v\rangle}{T^{2}}$ as a function of temperature for various fusion reactions. (Adapted from [6].)

we have thus to realize a temperature of about 150 million degrees (the temperature of a Maxwellian gas with an average energy of $1 \mathrm{eV}$ corresponds to $11600 \mathrm{~K}$ ), which is 10 times larger than in the Sun's core. In other words, the realisation of nuclear fusion on Earth requires the creation of a small but very hot Sun. Two questions then immediately arise: how does one realise such extreme temperatures and - equally important - how can one confine a gas at such high temperatures in a safe way?

\section{2. - Plasma confinement}

Two radically different approaches have been followed towards the realisation of nuclear fusion on Earth: inertial and magnetic confinement. In the first approach, lasers or particle beams are used to rapidly compress the nuclear fuel in order to achieve the conditions required for fusion. To this end, a small sphere with a diameter of a few hundred $\mu \mathrm{m}$ is filled with equal amounts of deuterium and tritium. Powerful laser or particle beams irradiate the surface of this little sphere as uniformly as possible, for about a billionth of a second. The outermost layers of the sphere vaporise nearly imme- 


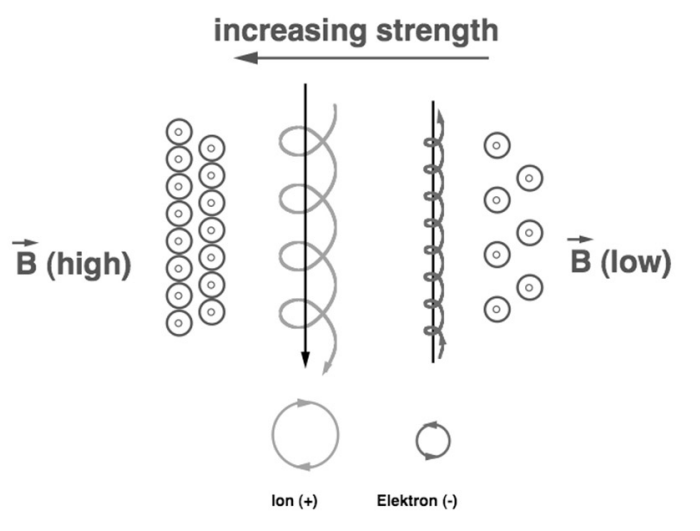

a)

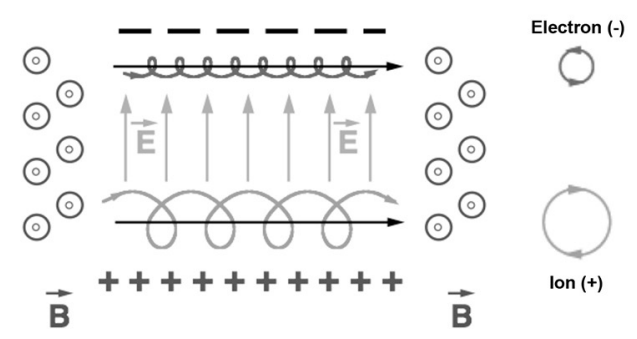

b)

Fig. 6. - Illustration of the effect of the inhomogeneity of the toroidal magnetic field on plasma confinement. The effect can be easily explained in two steps considering the formula for the Larmor radius: $\rho=\frac{m v_{\perp}}{q B}$. For the first step see a) at the left: formation of the electric field due to the inhomogeneity of the toroidal field. The figure shows a cross-section of the plasma; the orbits of ions and electrons are no longer perfectly circular, but the particles spiral vertically up and down: indeed from the formula follows that $\rho$ decreases as $B$ increases and increases as $B$ decreases. As ions and electrons rotate in opposite directions, they move vertically in opposite directions, creating an electric field in the plasma. Second step, see b) at the right: effect of the created electric field on the plasma particles. We consider a small section of the previous figure, such that $B$ is to a good approximation homogeneous over the section, and thus the effect of $E$ alone can be studied. As $B$ is constant over this section, the only parameter influencing the movement of the particles is the velocity. Positive ions are accelerated towards the negative layer of charges and decelerated towards the positive layer of charges, leading to an increase resp. decrease of the velocity of the particle. From the formula follows that with increasing velocity the Larmor radius decreases and decreases with increasing velocity: thus the particle spirals vertically outwards. A similar reasoning for the electrons leads to exactly the same result. Both electrons and ions move thus horizontally outward: the plasma is unstable. To solve this problem, the formation of the electric field has thus to be avoided. Adding a poloidal component to the toroidal field is a way to do this.

diately, generating an inward-propagating spherical shock wave, which compresses the sphere's contents enormously. At the end of this short compression phase, a density of a thousand times that of water is reached at a temperature of some tens of millions of degrees centigrade. Under these conditions, the deuterium and tritium nuclei start to fuse. The energy released by these first fusion reactions further heats the rest of the strongly compressed fuel, allowing fusion reactions to spread throughout the sphere.

The second approach is by magnetic confinement. Since this has proven to be the most successful method to date, magnetic fusion research is under development in laboratories all over Europe and throughout the world.

At the high temperatures necessary for nuclear fusion, atoms break up into their constituents, electrons and nuclei, and the fuel becomes fully ionised, a plasma. To 


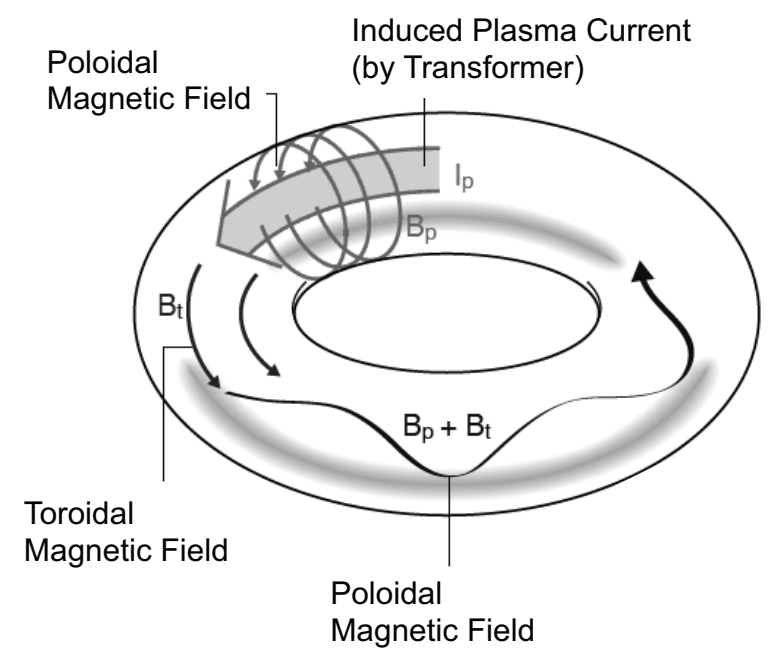

Fig. 7. - Principle of the tokamak: A large current induced in the plasma ring generates a poloidal magnetic field. The combination of this poloidal field with the main toroidal field results in a helical magnetic field, necessary for a stable confinement of the plasma in a toroidal configuration.

confine a hot plasma one can make use of magnetic fields, since charged particles will follow a helical path around the field lines owing to the Lorentz force. Possible movements perpendicular to the field are in this way highly restricted.

The most obvious way to realise this is a purely cylindrical configuration, which, however, suffers from plasma losses at both ends. These losses can be avoided by bending the magnetic field lines onto themselves, leading to a doughnut-shaped or toroidal magnetic field configuration. A toroidal magnetic field, however, is not homogeneous. Its curvature destroys most of the nice confinement properties of a purely cylindrical configuration: the magnetic field is largest near the axis of the torus, and decreases towards the outboard side. As a result, ions and electrons move in opposite vertical directions (fig. 6), giving rise to an electric field which, when combined with the toroidal magnetic field, expels all particles to the outside, irrespective of their charge. Without special measures, it is thus impossible to confine a toroidal plasma in a stable way. However, if the magnetic field can be given an extra twist, vertical drifts can be cancelled and a suitable trap can be made for fusion plasmas. This extra twist can be produced by means of electrical currents flowing inside or outside of the plasma ring. The tokamak concept relies on currents produced inside the plasma, whereas the stellarator concept relies on currents external to the plasma.

In a tokamak, a set of coils placed around the doughnut-shaped plasma chamber produces the main toroidal magnetic field. The electrical current necessary for the stability of the plasma flows in the plasma ring, which serves as the (only) secondary winding of an enormous transformer. The induced current in the plasma ring generates a poloidal 


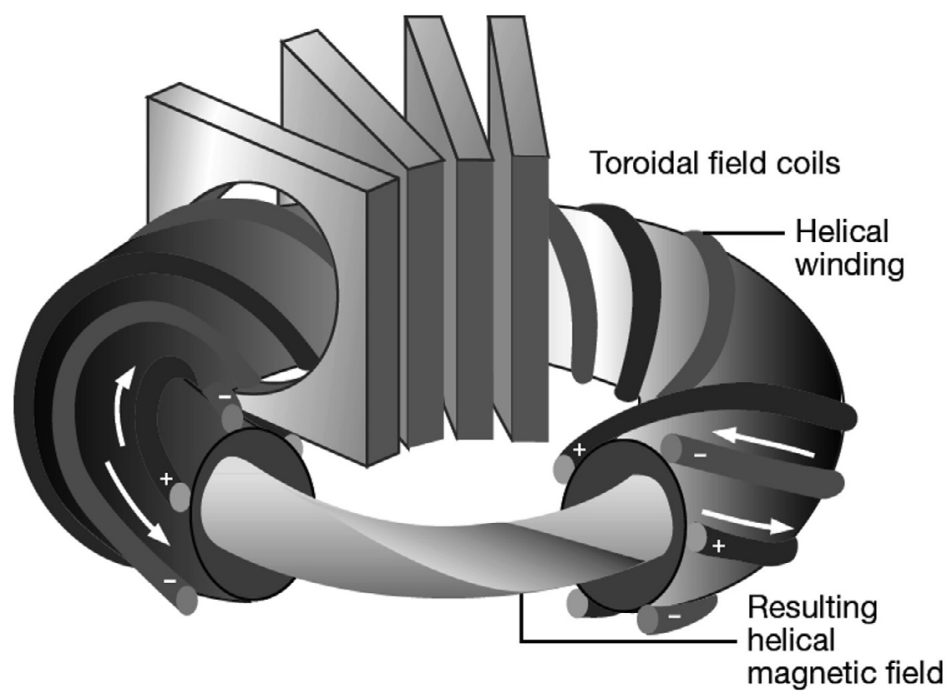

Fig. 8. - In a stellarator, helical coils external to the plasma ring create a supplementary magnetic field that, in combination with the main toroidal magnetic field, confines the plasma.

magnetic field. The combination of this poloidal field with the main toroidal field results in a helical magnetic field (fig. 7). The magnetic structure thus generated consists of an infinite set of nested toroidal magnetic surfaces, each with a slightly different twist, reducing further the leakage of particles and heat from the plasma. On each of these surfaces, the plasma pressure is constant. Tokamak research started in the Kurchatov Institute in Moscow under the leadership of Lev Artsimovich. The success of this configuration became at once clear at the Novosibirsk IAEA conference of 1968 where he announced that much higher temperatures could be obtained in a tokamak than all other magnetic configurations under study at that time.

The tokamak is however a pulsed device, since the transformer needs a steadily increasing current in its primary winding to induce the necessary plasma current.

Continuous operation of a fusion device can be obtained if the need for at transformer to induce the plasma current could be avoided. The stellarator is such a device, and it relies on currents external to the plasma to create the needed helical magnetic configuration. In its basic configuration, extra helical coils around the toroidal plasma provide the necessary additional twist to the toroidal magnetic field generated by the main field coils (fig. 8). These helical windings around the plasma ring, however, complicate the construction. In addition such "classical" stellarator configurations lack good confinement properties. Modern stellarators have optimised confinement properties, and are equipped with a set of coils with a complex shape that is determined numerically (fig. 9). Several devices of the stellarator type are in operation or in construction at this moment all over the world. The largest stellarator in the world that recently started operations (2016) is Wendelstein 7-X, located in Greifswald, Germany (fig. 10). The first experimental cam- 


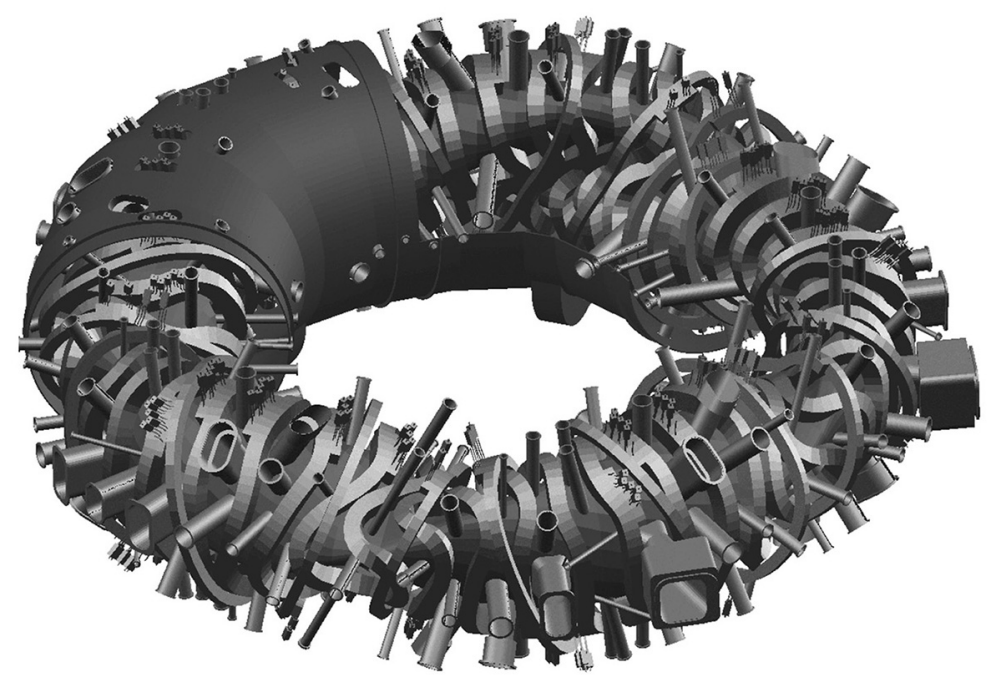

Fig. 9. - An illustration of the complex shapes of the coils of a modern stellarator, which combine the functions of the separate coil systems of fig. 8.

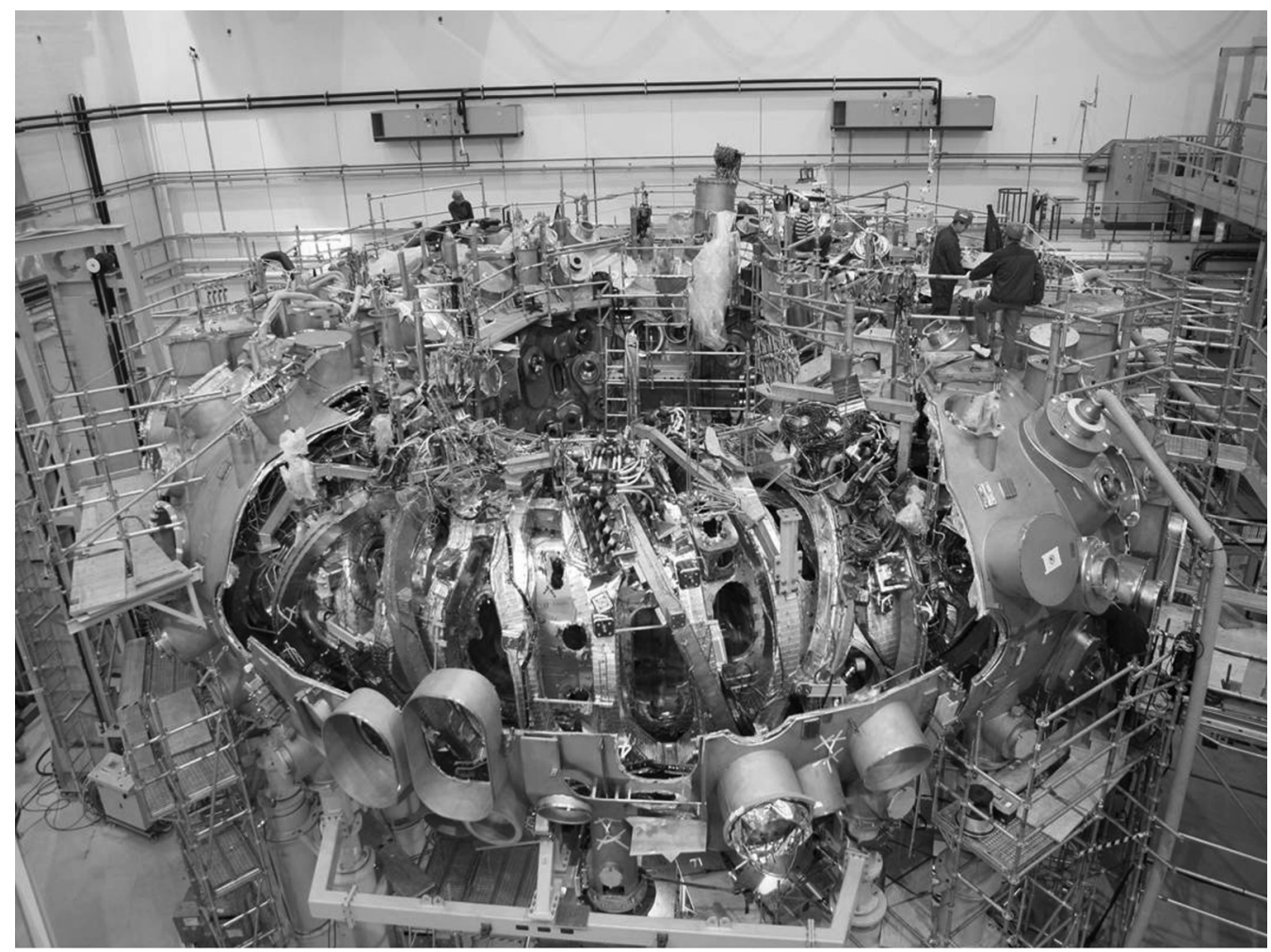

Fig. 10. - A picture of the stellarator Wendelstein 7-X, at the time of construction in November 2011, and now in operation since 2016 in Greifswald, Germany. 
paign showed encouraging results [7]. The device will be further upgraded in the coming years and in its final configuration will be able to run plasma pulses of 30 minutes. The largest stellarator-like device outside Europe is the LHD (Large Helical Device) of the National Institute for Fusion Science (NIFS, near Nagoya, Japan) that started operations in March 1998. Also this device has led to several rather important contributions [8].

\section{3. - Plasma heating}

The plasma current, needed to create the helical magnetic field providing stable confinement at the same time heats the plasma. Since the heating results from the finite resistance of the plasma, just as in an electrical heating element, this is referred to as ohmic heating. The plasma resistance, however, decreases with increasing plasma temperature, and at some millions of degrees, the efficiency of ohmic heating becomes too low to be useful for further heating. In fact, at these temperatures the plasma becomes a better conductor than copper at room temperature. That is why auxiliary heating methods have to be used to reach the temperatures required for fusion.

One additional heating method consists of injecting energetic particle beams of neutral hydrogen or deuterium into the plasma. This is possible because only neutral particles can penetrate the magnetic fields needed to confine the plasma. To this end, ions are created in a plasma source, accelerated with voltages up to 150000 volts and sent through a cloud of neutral gas. The accelerated ions "steal" electrons from atoms in this neutral gas cloud, and become energetic neutral particles, which in turn then enter the hot tokamak plasma without impediment from the confining magnetic fields of the fusion device. Once in the hot plasma, they are immediately ionised again, thus confined, and deposit their energy via collisions to the rest of the plasma particles. Powers of up to several million watts per neutral injector can be delivered to the plasma using this technique.

A second heating method sends electromagnetic waves into the plasma, by antennas or wave guides at the plasma edge. The principle underlying this method is similar to that of a microwave oven: the energy from the waves is most easily absorbed if the frequency used is equal to a "natural" frequency of the particles to be heated. Using the cyclotron frequency with which the charged plasma particles gyrate around the magnetic field lines, is an evident choice. Thus, two different heating systems exist: Ion Cyclotron Resonance Heating (ICRH) and Electron Cyclotron Resonance Heating (ECRH), depending on whether ions or electrons are heated. Ion cyclotron frequencies are in the $\mathrm{MHz}$ range $(20 \mathrm{MHz}$ and upwards), while electron cyclotron frequencies are approximately a 1000 times higher (at frequencies in the range of $100-200 \mathrm{GHz}$ ), due to the smaller mass of the electrons. Heating powers for high-frequency systems range from $100 \mathrm{~kW}$ to several tens of MW.

\section{4. - Characterizing the fusion reactivity of plasmas}

The power amplification factor $Q$ is defined as the ratio of the power produced by fusion reactions to the total heating power supplied externally. Two important milestones 
for the value of $Q$ are customarily used in fusion research. The first, breakeven, is reached when the heating power is equal to the power produced from fusion reactions, corresponding to a $Q$ value of one. The second, ignition, is reached when the additional heating systems can be switched off and the heat of the fusion reactions alone is sufficient to maintain the high temperatures needed for fusion. This corresponds to an infinite value for $Q$.

\section{5. - Progress in recent years}

The progress obtained in fusion research in the past three decades is clearly visible from the successively obtained values for the fusion product. Compared to the first fusion experiments, this value has increased by a factor of more than 10000 ! The results obtained on the largest tokamak in the world, JET (Joint European Torus, located at Culham Labs, close to Oxford, UK), have contributed significantly to this progress, and to date, values close to breakeven have been obtained in D-T experiments on this device. An important milestone was reached in 1991 with the first production of MWs of fusion power from controlled fusion reactions, as tritium was used for the first time as fuel in a tokamak [9]. These experiments, obtained with a mixture of $90 \% \mathrm{D}$ and $10 \% \mathrm{~T}$, generated fusion powers in the megawatt range for nearly 2 seconds, with a maximum of about $1.7 \mathrm{MW}$, corresponding to a $Q$ value of about 0.15 .

Further successes were obtained early in 1994 in the American tokamak TFTR (Tokamak Fusion Test Reactor) at the Plasma Physics Laboratory of Princeton University. In plasmas consisting of a mixture of $50 \%$ deuterium and $50 \%$ tritium, multi-megawatt level fusion powers were generated for about one second, with a maximum of $6.3 \mathrm{MW}$ [10]. Plasma temperatures in excess of 300 million degrees were reached in the plasma centre, 20 to 30 times hotter than in the centre of the Sun! In November 1994, fusion powers of more than $10 \mathrm{MW}$ were generated, corresponding to $Q$ values of about 0.27 [11].

Other record values were reached in 1996 on the Japanese Tokamak JT-60U of the Naka Fusion Research Establishment, located $150 \mathrm{~km}$ north of Tokyo, a division of the Japanese Atomic Energy Research Institute (JAERI). This machine demonstrated temperatures in excess of 520 million degrees [12], the highest temperature ever realised by man on Earth. Even more important, a record value for the fusion triple product was obtained in pure deuterium plasmas. If the same conditions had been realised in deuterium-tritium plasmas, it would have resulted in a $Q$ value of about 1.25 , i.e. conditions better than breakeven [13].

The most impressive results in fusion research up to now were obtained in the JET, in October and November 1997. Experiments in 50\% D-50\% T plasmas resulted in over $16 \mathrm{MW}$ of fusion power during about 1 second, with $Q$ values in excess of 0.65 [14]. These are the highest fusion powers and $Q$ values ever reached in D-T reactions, thereby effectively resulting in the first demonstration of breakeven in reactor grade $\mathrm{D}-\mathrm{T}$ fusion plasmas. A quasi steady-state generation of fusion power has also been demonstrated: over $4 \mathrm{MW}$ of fusion power were produced for time intervals of more than 5 seconds [15], a duration only limited by the actual technical constraints of JET. 


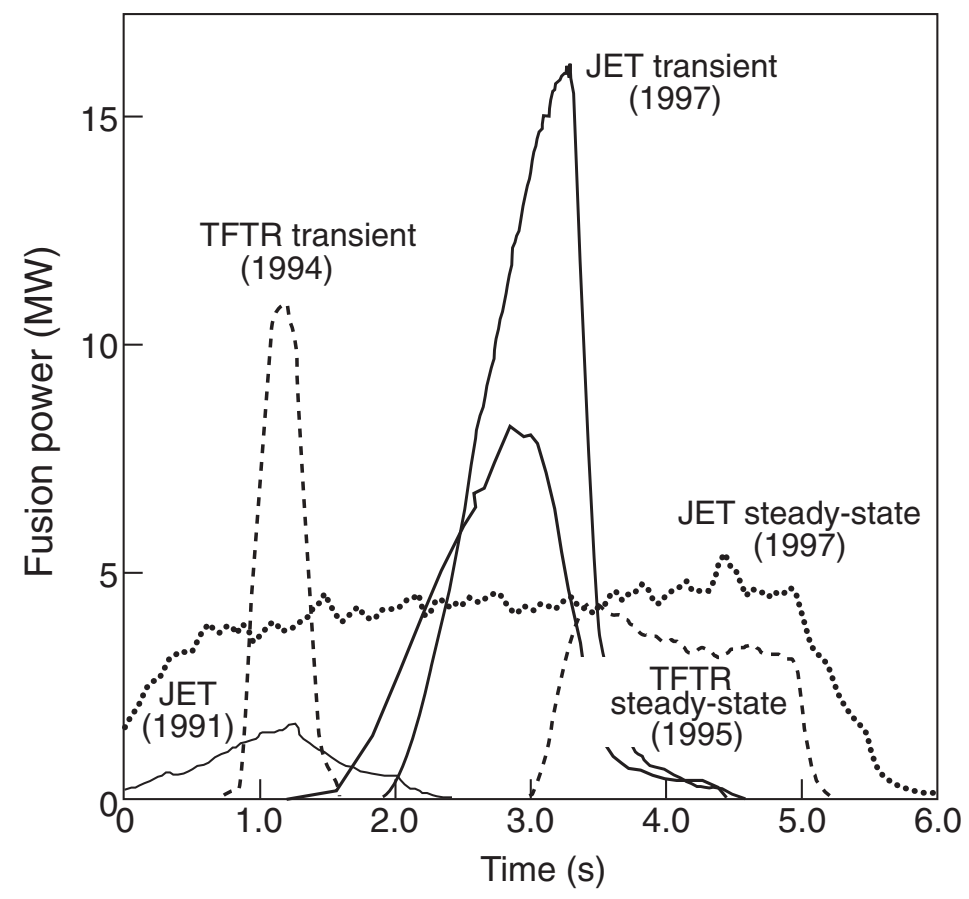

Fig. 11. - Time traces of the fusion power released in different high-performance deuteriumtritium fusion experiments in TFTR and JET. (Adapted from [15].)

A summary of the different high performance D-T pulses obtained on JET and TFTR, as discussed above, is presented in fig. 11 .

The success of the D-T experiments on JET and TFTR proves the scientific feasibility of controlled nuclear fusion. In addition, those experiments also allowed to get a first glimpse of the effects on the plasma of fast alpha particles.

\section{6. - Conditions for a fusion reactor: Lawson criterion}

The Lawson criterion determines conditions for an operating reactor. In its simplest form it can be defined as follows: the sum of the power densities from auxiliary heating $P_{\text {heat }}$ and $\alpha$ particles has to be at least equal to the power densities lost by radiation $P_{\text {radiation }}$ and transport, $P_{\text {transport }}$ (convection, conduction). This can be written as

$$
P_{\text {heat }}+P_{\alpha} \geq P_{\text {transport }}+P_{\text {radiation }}
$$

To simplify the calculations, we consider in what follows a pure D-T plasma without impurities (i.e. effective charge of the plasma $Z_{\text {eff }}=1$ ); this implies also that we neglect the presence of $\mathrm{He}$ ash from the reaction. We assume only Bremsstrahlung losses for $P_{\text {radiation }}$ (given by the formula $P_{\text {Brems }}\left(\right.$ in $\mathrm{W} / \mathrm{m}^{3}$ ) $=C_{B} T^{1 / 2} n^{2}$ if $T$ is expressed in 
$\mathrm{keV}$, and $n$ in $\mathrm{m}^{-3}$ with $\left.C_{B}=5.35 \times 10^{-37} \mathrm{~W} \mathrm{~m}^{3} \mathrm{keV}^{-1 / 2}[16]\right)$ and we characterize all transport-related energy losses by the term $P_{\text {transport }}$, making use of the confinement time $\tau_{\mathrm{E}}$, defined by the relation $\mathrm{d} W_{\mathrm{p}} / \mathrm{d} t=W_{\mathrm{p}} / \tau_{\mathrm{E}}$, where $W_{\mathrm{p}}$ is the energy density in the plasma. We also take into account that

$$
P_{\text {heat }} Q=P_{\text {fusion }}=5 P_{\alpha} \text {. }
$$

And that the total plasma energy density is the sum of the ion and electron energy densities:

$$
W_{p}=\frac{3}{2}\left(n_{e} k T_{e}+n_{i} k T_{i}\right)
$$

with $T_{i}$ and $T_{e}$ in $\mathrm{K}, n_{e}$ and $n_{i}$ in $\mathrm{m}^{-3}$, and $k=1.38 \times 10^{-23} \mathrm{~J} / \mathrm{K}$ the Boltzmann constant.

In a neutral D-T plasma electron and ion densities are the same $n_{e}=n_{i}=n$; we also assume that the plasma is in thermal equilibrium i.e. there is no difference between electron and ion temperatures $T_{e}=T_{i}=T$. Therefore

$$
W_{p}=3 n k T \text {. }
$$

Assuming a pure $50 \% \mathrm{D}-50 \% \mathrm{~T}$ plasma leads also to $n_{T}=n_{D}=1 / 2 n_{e}=1 / 2 n$. The expression for the power density delivered to the plasma by the $\alpha$ particles, can thus be written as

$$
P_{\alpha}=\frac{1}{4} n_{D} n_{T} E_{\alpha}\langle\sigma v\rangle=\frac{1}{4} n^{2} E_{\alpha}\langle\sigma v\rangle
$$

Expressing $E_{\alpha}$ and $T_{k}$ in $\mathrm{keV},(11)$ can be written as

$$
\frac{1}{4} n^{2} E_{\alpha}\langle\sigma v\rangle\left(\frac{Q+5}{Q}\right) \geq \frac{3 n T_{k}}{\tau_{E}}+C_{B}^{*} Z_{\mathrm{eff}} T_{k}^{1 / 2} n^{2}
$$

And finally

$$
n \tau_{E} \geq \frac{3 T_{k}}{\frac{\langle\sigma v\rangle E_{\alpha}(Q+5)}{4 Q}-C_{B}^{*} Z_{\mathrm{eff}} T_{k}^{1 / 2}}
$$

where $C_{B}^{*}=C_{B} / 1.602 \times 10^{-16}=3.34 \times 10^{-21} \mathrm{~W} \mathrm{~m}^{3} \mathrm{keV}^{-1 / 2}$.

The right-hand side formula shows a minimum for about $T_{k}=25 \mathrm{keV}$. Approximating $\langle\sigma v\rangle\left[\mathrm{in} \mathrm{m}^{-3} \mathrm{~s}\right]=1.1 \times 10^{-24} T_{k}^{2}$ (valid to within $15 \%$ in the interval $10 \mathrm{keV}<T_{k}<25 \mathrm{keV}$ ) and setting $Z_{\text {eff }}=1$ (pure D-T plasma) we find

$$
\text { for breakeven }(Q=1): n \tau_{E} \geq 2.5 \times 10^{19} \mathrm{~m}^{-3} \mathrm{~s}
$$


and

$$
\text { for ignition }(Q=\infty): n \tau_{E} \geq 1.5 \times 10^{20} \mathrm{~m}^{-3} \mathrm{~s} \text {. }
$$

An alternative formulation of the Lawson criterion is in the form of the so-called fusion triple product. Considering again only the temperature range where the minimum of eq. (17) occurs $\left(k T_{e} \sim 10-25 \mathrm{keV}\right)$, we can neglect to a first approximation the corrections for the Bremsstrahlung losses. Thus we find, with the same conventions for the various physical quantities as in eq. (17):

$$
n \tau_{E} T_{k} \geq \frac{12 Q T_{k}^{2}}{(Q+5)\langle\sigma v\rangle E_{\alpha}} .
$$

The minimum for the right-hand side is obtained at the maximum for $\langle\sigma v\rangle / T_{k}^{2}$, as shown above, about $T_{k}=13 \mathrm{keV}$. We thus find

$$
\text { for breakeven }(Q=1): n \tau_{E} T_{k} \geq 5 \times 10^{20} \mathrm{~m}^{-3} \mathrm{~s} \mathrm{keV}
$$

and

$$
\text { for ignition }(Q=\infty): n \tau_{E} T_{k} \geq 3 \times 10^{21} \mathrm{~m}^{-3} \mathrm{~s} \mathrm{keV} \text {. }
$$

These simplified expressions should give a good idea of the physics behind the famous Lawson criterion. For a more detailed treatment, taking into account radiation, impurities etc. we refer to the interesting paper by Rebhan and Van Oost [17].

\section{7. - Future prospects: JET as a testbed for ITER, JT-60SA, ITER and DEMO}

7'1. JET as a testbed for ITER. - JET is currently focused on providing experimental evidence to questions raised by the ITER team. Because of its worldwide unique capabilities (able to operate with both beryllium as wall material and tritium) and its large size, JET is ideally placed to do so. JET's "Programme in Support of ITER" has been elaborated along three main axes: i) experimentation with an ITER-like wall (combination of $\mathrm{W}$ and $\mathrm{Be}$ as a first wall); ii) development of plasma configurations and parameters towards the most ITER-relevant conditions achievable today and iii) integrated experimentation in deuterium-tritium. The JET auxiliary heating systems are upgraded with currently $45 \mathrm{MW}$ of installed heating power, allowing access to ITER-relevant operational conditions. This will provide access to conditions of melt layer formation both on the beryllium first wall and the tungsten divertor and, help to make further progress in hybrid and advanced scenarios for ITER, which require full or partial current profile control, thereby making use of new dedicated diagnostics. Results from the first years of 
TABLE II. - Main parameters of important tokamaks.

\begin{tabular}{|c|c|c|c|c|c|}
\hline & $\mathrm{T}-10$ & DIII-D & JT-60SA & JET & ITER \\
\hline \multirow[t]{2}{*}{ Land/Organisation } & Russian & USA & Japan & GB/ & International \\
\hline & Federation & & & EURATOM & \\
\hline Plasma shape & circular & elliptical (D) & elliptical (D) & elliptical (D) & elliptical (D) \\
\hline \multirow[t]{2}{*}{ Minor radius (m) } & 0.3 & 0.67 (hor) & 1.18 (hor) & 1.25 (hor) & 2.0 (hor) \\
\hline & & 1.74 (vert) & 2.30 (vert) & 2.1 (vert) & 3.7 (vert) \\
\hline Major Radius (m) & 1.5 & 1.67 & 2.96 & 2.96 & 6.2 \\
\hline \multicolumn{6}{|l|}{ Toroidal } \\
\hline Magnetic field (T) & 2.5 & 2.2 & 2.25 & 3.5 & 5.3 \\
\hline \multicolumn{6}{|l|}{ Plasma } \\
\hline Current (MA) & 0.7 & 3.0 & 5.5 & 5.0 & $15(17)$ \\
\hline Pulse length (s) & 4 & 10 & 100 & 60 & $300-500$ \\
\hline \multicolumn{6}{|l|}{ Injection of } \\
\hline \multicolumn{6}{|l|}{ Neutral particle } \\
\hline \multirow[t]{3}{*}{ beams (MW) } & - & 20 & 34 & 30 & \\
\hline & & & & & $73(130)$ \\
\hline & & & & & in total \\
\hline $\begin{array}{l}\text { Injection of } \\
\text { electromagnetic }\end{array}$ & & & & & \\
\hline waves (MW) & 1.5 & 8 & 7 & 38 & \\
\hline
\end{tabular}

operations with the ITER like wall are summarized in [18]. Preparations for a new D-T campaign are currently underway in JET.

7'2. JT-60SA. - In February 2007, Euratom and Japan signed the Broader Approach agreement. This aims to complement the ITER Project and to accelerate the realisation of fusion energy by carrying out R\&D and developing some advanced technologies for future demonstration fusion power reactors (DEMO). Within the Broader Approach, three main projects are being implemented: i) the construction in Japan of the large superconducting tokamak JT-60SA aimed at developing operational scenarios relevant for ITER (International Thermonuclear Experimental Reactor) and DEMO; ii) the construction of facilities to test components to be adopted in the research machine IFMIF (International Fusion Material Irradiation Facility) aimed to study the material damage caused by high-energy neutron flux generated in fusion reactions, the construction of a prototype of the liquid lithium IFMIF target; iii) the creation of the International Fusion Energy Research Centre (IFERC) including a centre for advanced materials development and a supercomputing centre [19].

Parameters for JT-60SA are summarized in table II. JT-60SA is a fusion experiment designed to support the operation of ITER and to investigate how best to optimise the 
operation of fusion power plants that are built after ITER. It is a joint international research and development project involving Japan and Europe, and is to be built in Naka, Japan using infrastructure of the former JT-60 Upgrade experiment, for which we cited several important experimental results in sect. 5 above. SA stands for "super, advanced", since the experiment will have superconducting coils and study advanced modes of plasma operation. According to the current planning, first plasmas on JT60SA are foreseen in 2019. More information on this interesting future tokamak can be found in [20].

7.3. ITER. - The results summarized in sect. 5 provide crucial information for the design of a next large tokamak, aimed at demonstrating the technical feasibility for largescale energy production. This next step is ITER, short for International Thermonuclear Experimental Reactor, currently under construction in Cadarache, France as a combined effort between Europe, Japan, the Russian Federation, South Korea, India, China and the United States. This device will thus for the first time in history allow mankind to produce hundreds of MW of fusion power from nuclear fusion reactions at temperatures over 100 million degrees. ITER is expected to generate fusion powers of the order of $500 \mathrm{MW}$ in pulses of $300-500$ seconds. Specifications for ITER (and a few other major tokamaks) are summarised in table II. After ITER, the construction of a demonstration reactor, currently termed DEMO, is foreseen, which should show not only the technical, but also the economical feasibility of fusion.

7`4. DEMO. - Before DEMO can become a practical demonstration of electricity generation on a power-plant scale that satisfies various socio-economic goals, major challenges must be resolved. Although ITER will bring significant advances, there remains a large gap between ITER and DEMO. Main differences between ITER and DEMO are summarized in table III.

DEMO is currently based on the tokamak, as this is the most advanced fusion concept to date. Reactor studies are also being developed for Helical Devices (see, e.g., [21-24]). However, a decision on a next step stellarator/helical device can only take place when the main results of the current large helical devices in operation or construction have been obtained.

To lead a coordinated effort in the EU towards DEMO, the Power Plant Physics and Technology Department (PPP\&T) has been established in 2011 [25]. The aims of the DEMO studies in Europe are: i) to quantify key physics and technology prerequisites for DEMO; ii) to identify the most urgent technical issues that need to be solved in physics and technology and iii) to plan and implement supporting physics and technology R\&D.

Two DEMO design options are currently being investigated by PPP\&T. (See table IV for main characteristics):

i) DEMO Model 1: A "conservative baseline design" that could be delivered in the short to medium term, based on the expected performance of ITER with reasonable 
TABLE III. - Main differences between ITER and DEMO.

\begin{tabular}{|l|l|}
\hline ITER & DEMO \\
\hline Experimental Device & Close to commercial plant \\
\hline 400s pulses & $\begin{array}{l}\text { Long pulses, high duty cycle or } \\
\text { steady state }\end{array}$ \\
\hline Long interpulse time & Minimum set of diagnostics only \\
Many diagnostics & needed for operations \\
\hline Many H\&CD systems & Reduced set of H\&CD systems \\
\hline No T breeding required & Self-sufficient T breeding \\
\hline 316 SS structural material & Reduced activation structural \\
& material \\
\hline Low n-fluence, low dpa & High n-fluence, high dpa \\
Low material damage & Significant material damage \\
\hline
\end{tabular}

TABLE IV. - Main parameters of the early DEMO 1 and more advanced DEMO 2 model currently under investigation by the PPPEST Department of EFDA. Shown are the thermal output power $\left(P_{\mathrm{th}}\right)$, the net electrical power to the grid $\left(P_{\mathrm{net}}\right)$, the recirculating power $\left(P_{\mathrm{rec}}\right)$, the auxiliary heating power $\left(P_{\text {aux }}\right)$, major radius $\left(R_{0}\right)$ and minor radius $(a)$ of the device, plasma current $\left(I_{\mathrm{p}}\right)$ and toroidal magnetic field on axis $\left(B_{\mathrm{t}}\right)$.

\begin{tabular}{|l|l|l|}
\hline $\begin{array}{l}\text { Device } \\
\text { Operation Mode }\end{array}$ & $\begin{array}{l}\text { DEMO 1 } \\
\text { Pulsed }\end{array}$ & $\begin{array}{l}\text { DEMO 2 } \\
\text { Steady State }\end{array}$ \\
\hline$P_{\text {th }}(\mathrm{MW})$ & 2200 & 2700 \\
\hline$P_{\text {net }}(\mathrm{MW})$ & 500 & 500 \\
\hline$P_{\text {rec }}(\mathrm{MW})$ & 594 & 600 \\
\hline$P_{\text {aux }}(\mathrm{MW})$ & 50 & 350 \\
\hline$R_{0}(\mathrm{~m})$ & 9.0 & 8.15 \\
\hline$a(\mathrm{~m})$ & 2.25 & 3.0 \\
\hline$I_{\mathrm{p}}(\mathrm{MA})$ & 14.1 & 19.8 \\
\hline$B_{\mathrm{t}}(\mathrm{T})$ on axis & 6.8 & 5.0 \\
\hline
\end{tabular}


improvements in science and technology i.e. a large, modest power density, long-pulse inductively supported plasma in a conventional plasma scenario.

ii) DEMO Model 2: an "optimistic design" based upon more advanced assumptions which are at the upper limit of what may be achieved, leading to a steady state plasma scenario where a large fraction of the plasma current is induced non-inductively, i.e. without making use of the transformer. This is currently a rather speculative option.

From table III there follows immediately a minimum set of research topics that need to be (much) further developed: heating systems capable of heating the large plasmas foreseen for DEMO, diagnostic systems that can sustain the heavy neutron loading, tritium-generating modules (so-called tritium blankets) to foresee tritium self-sufficiency, and new materials that will be compatible with the high heat and neutron loads. This implies also that new laboratories will have to be built to be able to explore possibilities and further develop existing systems. Among others, a material research facility to study the behaviour of various candidate materials under a flux of $14.1 \mathrm{MeV}$ neutrons (preparations for such a facility, IFMIF, are already underway in Japan and Europe [26]), a facility to develop, e.g., Neutral Beam Heating systems (if these are viable at all for DEMO) with acceleration energies between 1 and $2 \mathrm{MeV}$, based on negative ion sources (in contrast to the positive ion sources used currently) etc. A first step in this direction is the PRIMA facility being built in Padova, Italy for the development of the ITER NBI systems [27]. But more efforts in various other fields will have to follow, and this will unavoidably take time.

The European Fusion Development Agreement (EFDA) has released in November 2012 a roadmap for the realization of fusion electricity to the grid by 2050 [28]. This roadmap covers three periods: i) the upcoming European Research Framework Programme, Horizon 2020, ii) the years 2021-2030 and iii) the period 2031-2050. ITER is the key facility of the roadmap as it is expected to achieve most of the important milestones on the path to fusion power. The second period is focused on maximizing ITER exploitation and on preparing the construction of DEMO. Building and operating DEMO is the subject of the last roadmap phase (time horizon about 2050).

\section{8. - Will fusion be a safe, clean and inexhaustible energy source?}

The concept of a future fusion power device based on a tokamak is outlined in fig. 12 . Due to the high reactivity of the $\mathrm{D}-\mathrm{T}$ fusion reaction, deuterium and tritium will most likely be selected as fuel for the first generation of fusion power stations. The electrically charged $\alpha$-particles from fusion reactions are confined by the magnetic field. These highenergy helium nuclei collide with the background plasma particles, i.e. with the $\mathrm{D}$ and $\mathrm{T}$ ions in the plasma and maintain the temperature rendering additional heating systems quasi unnecessary as soon as ignition is reached.

In contrast to the $\alpha$-particles, the neutrons generated by the $\mathrm{D}$ - $\mathrm{T}$ reaction have no electrical charge and escape immediately from the confining magnetic field in the plasma chamber. These neutrons are not lost, but serve a dual purpose in the reactor. First, 


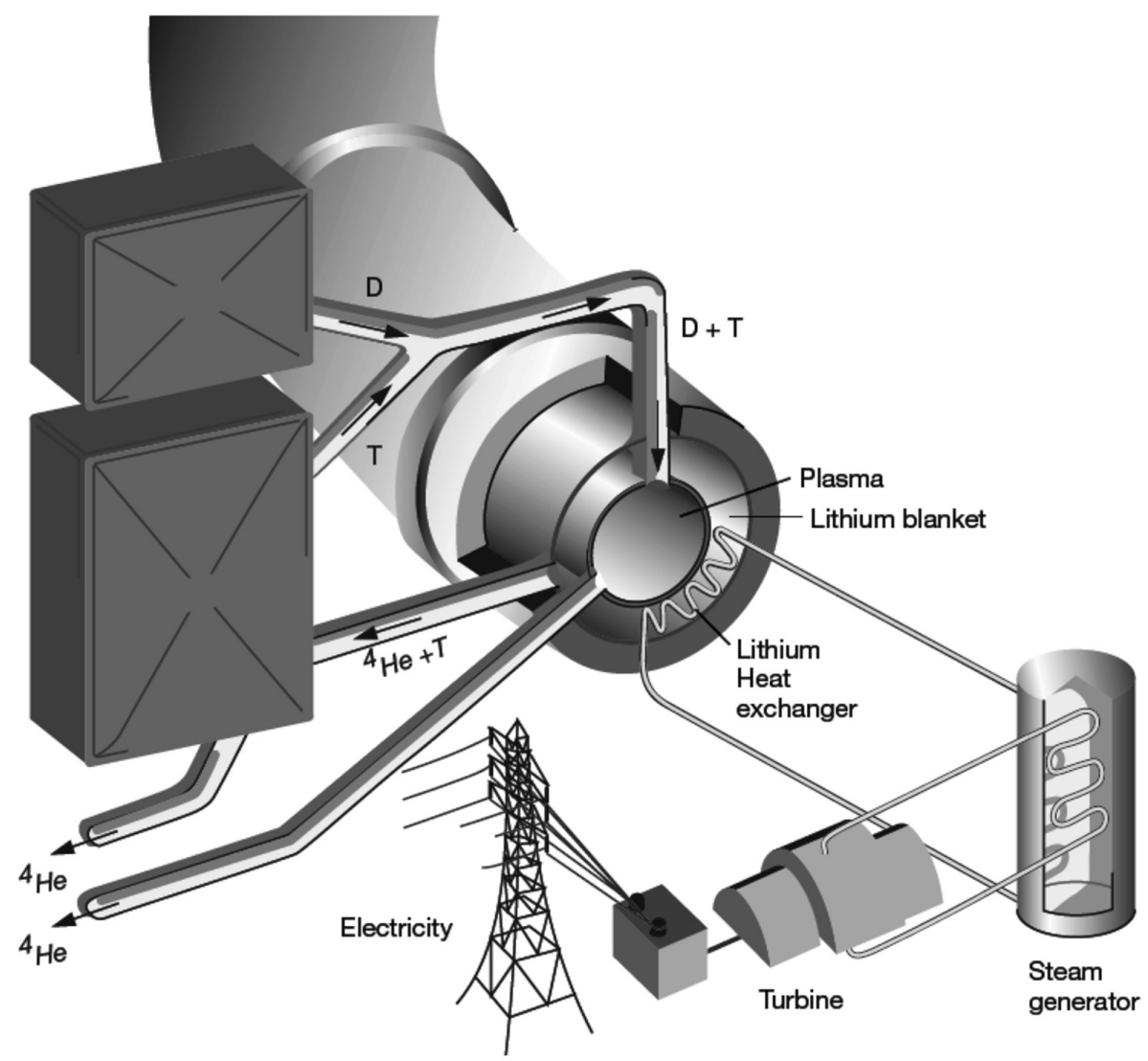

Fig. 12. - Many obstacles still have to be overcome before the first nuclear fusion power plant will be put into operation. Eventually, heat generated in fusion reactions will be used to generate steam to drive a turbine, with electrical power as final result.

they produce tritium in a blanket surrounding the reactor containing lithium or lithium compounds, according to the reactions given in table V. This is necessary to refuel the plasma with tritium, consumed in fusion reactions. Second, the energy of the neutrons deposited in the blanket is converted into heat, which is in turn is transported to the exterior by the cooling system and used to generate steam and drive classical turbines for the production of electricity.

The main advantage of fusion is the absence of any long-lived radioactive waste and its inherent safety. The quantity of fuel in the reaction chamber at any moment is only a few grams, in contrast to the presence of several tons of fuel in the core of a fission reactor. Furthermore, the fusion process is not susceptible to an uncontrolled release of energy, as it is in essence a "gas burner" and thus can be halted in a very short time by cutting the supply of fusion fuel. There is thus no risk of a runaway reaction, and Chernobyl-like accidents are absolutely excluded. 
TABLE V. - Tritium production from Lithium.

\begin{tabular}{|c|}
\hline${ }^{7} \mathrm{Li}+\mathrm{n} \rightarrow{ }^{4} \mathrm{He}+\mathrm{T}+\mathrm{n}-2.47 \mathrm{MeV}$ \\
\hline${ }^{6} \mathrm{Li}+\mathrm{n} \rightarrow{ }^{4} \mathrm{He}(2.05 \mathrm{MeV})+\mathrm{T}(2.73 \mathrm{MeV})$ \\
\hline
\end{tabular}

As previously mentioned, the fuels for the most accessible fusion reactions are deuterium and tritium. Deuterium can be extracted in large quantities from ordinary water found in rivers and oceans, rendering each country less dependent on fuel supply from other parts of the planet. Moreover, a minimal amount of fuel is necessary to cover the lifetime electricity needs of a European citizen: about $10 \mathrm{~g}$ of $\mathrm{D}$ and $15 \mathrm{~g}$ of $\mathrm{T}$ (and about twice this amount for a citizen of the United States). As explained above, tritium will be produced in situ from the irradiation of $\mathrm{Li}$ with the neutrons from the fusion reaction. Thus the real combustibles are in fact $\mathrm{D}$ and $\mathrm{Li}$, both of which are non-radioactive. World reserves of Li are sufficient for many thousand years of operation of D-T fusion power plants. Studies are underway to also extract it from seawater [29]. If at a later stage the D-D reaction could be used (requiring, however, even much higher plasma temperatures), we could have at our disposal a virtually unlimited energy source, as reserves of $\mathrm{D}$ are sufficient for several million years of energy production. In both cases, the reaction product is helium, which is chemically inert and non-radioactive. With nuclear fusion there is thus no release of gases which contribute to the greenhouse effect, acid rain or the depletion of the ozone layer. Moreover, helium is the only gas still liquid at temperatures down to absolute zero, thus offering the only possibility for cooling low temperature superconductors (as e.g. in modern MRI devices). It is thus not a "waste product" but in fact is essential for all applications involving ultra-low temperatures. This is especially important since helium is rather scarce on Earth.

The high-energy neutrons will activate the metallic reactor structure and hence make it radioactive. Low activation materials have to be selected to minimise this effect. In this way, the total radioactive inventory of a fusion reactor could be made one million times smaller than that of existing fission reactors. The activation problem could be drastically reduced in a distant future by the use of other fusion reactions, which liberate fewer neutrons. The radioactivity induced by the $\mathrm{D}^{3}{ }^{3} \mathrm{He}$ reaction for example, would be about 50 times lower.

The various steps that remain to be taken by thermonuclear fusion research indicate that some decades will still be needed to realise a practically useable energy source. The most recent results support the attractiveness of the fusion concept for energy production. A sustained effort - in laboratories worldwide - brings the vision of an energy supply by thermonuclear fusion ever closer to realisation. We all know that fusion is a challenging undertaking and that patience will be needed, but it is more than worth the effort given the difficulties we are facing in the future with our current energy supply and its suspected influence on climate. It will evidently be up to you, young researchers, to tackle these interesting problems. If successful, this will be your important contribution to the benefit of all people on Earth. A true challenge for an immense reward! 
Appendix A.

Atomic mass and binding energy per nucleon [30], stability and natural abundance for light nuclides. Note the remarkable absence of stable nuclides with mass number 5 and 8 .

\begin{tabular}{|c|c|c|c|c|}
\hline Nucleus & $\begin{array}{l}\text { Stable/ } \\
\text { Unstable } \\
\text { (half-life) }\end{array}$ & $\begin{array}{c}\text { Natural } \\
\text { Abundance }\end{array}$ & $\begin{array}{c}\text { Binding Energy per } \\
\text { nucleon }(\mathrm{keV})\end{array}$ & Atomic Mass (u) \\
\hline${ }^{1} \mathrm{H}$ & $\mathrm{s}$ & $99.985 \%$ & n.a & 1.0078250 \\
\hline $\mathrm{n}$ & $\mathrm{u}(12 \min )$ & n.a. & n.a & 1.0086649 \\
\hline${ }^{2} \mathrm{H}=\mathrm{D}$ & $\mathrm{s}$ & $0.015 \%$ & 1112.283 & 2.0141018 \\
\hline${ }^{3} \mathrm{H}=\mathrm{T}$ & $\mathrm{u}(12.32 \mathrm{yr})$ & n.a. & 2827.265 & 3.0160493 \\
\hline${ }^{3} \mathrm{He}$ & $\mathrm{s}$ & $0.000137 \%$ & 2572.680 & 3.0160293 \\
\hline${ }^{4} \mathrm{He}$ & $\mathrm{s}$ & $99.999863 \%$ & 7073.915 & 4.0026033 \\
\hline${ }^{6} \mathrm{Li}$ & s & $92.41 \%$ & 5332.331 & 6.0151229 \\
\hline${ }^{7} \mathrm{Li}$ & $\mathrm{s}$ & $7.59 \%$ & 5606.439 & 7.0160034 \\
\hline${ }^{9} \mathrm{Be}$ & s & $100 \%$ & 6462.668 & 9.0121831 \\
\hline${ }^{10} \mathrm{~B}$ & $\mathrm{~s}$ & $19.8 \%$ & 6475.083 & 10.0129369 \\
\hline${ }^{11} \mathrm{~B}$ & $\mathrm{~s}$ & $80.2 \%$ & 6927.732 & 11.0093052 \\
\hline${ }^{12} \mathrm{C}$ & $\mathrm{s}$ & $98.89 \%$ & 7680.144 & 12.0000000 \\
\hline${ }^{13} \mathrm{C}$ & $\mathrm{s}$ & $1.11 \%$ & 7469.849 & 13.0033548 \\
\hline${ }^{14} \mathrm{~N}$ & $\mathrm{~s}$ & $99.634 \%$ & 7475.614 & 14.0030740 \\
\hline${ }^{15} \mathrm{~N}$ & $\mathrm{~s}$ & $0.366 \%$ & 7699.460 & 15.0001089 \\
\hline${ }^{16} \mathrm{O}$ & $\mathrm{s}$ & $99.762 \%$ & 7976.206 & 15.9949146 \\
\hline${ }^{17} \mathrm{O}$ & $\mathrm{s}$ & $0.038 \%$ & 7750.728 & 16.9991318 \\
\hline${ }^{18} \mathrm{O}$ & $\mathrm{s}$ & $0.2 \%$ & 7767.097 & 17.9991596 \\
\hline${ }^{19} \mathrm{~F}$ & $\mathrm{~s}$ & $100 \%$ & 7779.018 & 18.9984032 \\
\hline${ }^{20} \mathrm{Ne}$ & $\mathrm{s}$ & $90.48 \%$ & 8032.240 & 19.9924402 \\
\hline${ }^{21} \mathrm{Ne}$ & $\mathrm{s}$ & $0.27 \%$ & 7971.713 & 20.9938467 \\
\hline${ }^{22} \mathrm{Ne}$ & $\mathrm{s}$ & $9.25 \%$ & 8080.465 & 21.9913851 \\
\hline
\end{tabular}




\section{Further Reading}

A recent overview of the status of fusion research (magnetic and inertial) can be found in the "Insight Section" (66 pages) of the May 2016 volume of Nature Physics (http://www . nature.com/nphys/insight/nuclear-fusion/index.html).

\section{REFERENCES}

[1] Lang and Kenneth R., in The Sun from space, 2nd edition (Springer Verlag, Berlin) 2009, ISBN-13: 978-3540769521, Chapt. 3.

[2] NACRE (Nuclear Astrophysics Compilation of Reaction Rates), http://pntpm.ulb.ac. be/nacre.htm; Angulo C. et al., Nucl. Phys. A, 656 (1999) 3.

[3] RAEDER J. et al., in Controlled Nuclear Fusion: Fundamentals of its Utilization for Energy Supply (John Wiley \& Sons, New York) 1986, ISBN-13: 978-047110312, Chapt. 1.

[4] Rebhan E., in Heisser als das Sonnenfeuer (Piper Verlag, München and Zürich) 1992, ISBN-13: 978-3492031097, Chapt. 5.

[5] Atzeni S. and Meyer-Ter-Vehn J., in The Physics of Inertial Fusion: Beam-Plasma Interaction, Hydrodynamics, Hot Dense Matter (Oxford University Press, Oxford), 2004, ISBN-13: 978-0198562641, Chapt. 1.

[6] Emmert G. A., El-Guebaly L. and Kulcinski G. L. et al., Nucl. Fusion, 29 (1989) 1427.

[7] Wolf R. et al., Nucl. Fusion, 57 (2017) 102020.

[8] Yamada H. et al., Nucl. Fusion, 51 (2011) 094021.

[9] The JET team, Nucl. Fusion, 32 (1992) 187.

[10] Strachan J., Adler H. and Alling P. et al., Phys. Rev. Lett., 72 (1994) 3526; Hawryluk R. J., Adler H. and Alling P. et al., Phys. Rev. Lett., 72 (1994) 3530.

[11] McGuire K. M., Adler H. and Alling P. et al., Phys. Plasmas, 2 (1995) 2176.

[12] Ishida S. et al., in Proceedings of the 16th IAEA Conference on Fusion Energy, Montreal Vol. 1 (IAEA, Vienna) 1997, p. 315.

[13] Fujita T., Kamada Y. and Ishida S. et al., Nucl. Fusion, 39 (1999) 1637.

[14] Keilhacker M., Gibson P. and Gormezano C. et al., Nucl. Fusion, 39 (1999) 209.

[15] JACQUinot J. and the JET Team, "Deuterium-tritium operation in magnetic confinement experiments: results and underlying physics", Plasma Phys. Control. Fusion, 41 (1999) A13.

[16] Wesson J., in Tokamaks, 3rd edition (Oxford University Press, Oxford, UK) 2004, ISBN 0-19-850922-7.

[17] Rebhan E. and Van Oost G., in Proceedings of the Sixth Carolus Magnus Euro-Summer School On Plasma And Fusion Energy Physics Brussels, Belgium, Fusion Sci. Technol., 45 (2004) 15.

[18] Litaudon X. et al., Nucl. Fusion, 57 (2017) 102001.

[19] Tsunematsu T., Fusion Eng. Des., 84 (2009) 122.

[20] Barabaschi P., Kamada Y. and Ishida S., in Proceedings of the 25th IAEA Conference on Fusion Energy, St-Petersburg, Russian Federation, 2014 (IAEA) 2014, paper OV/3.

[21] Beidler C. D. et al., Plasma Fusion Research Series, 5 (2002) 149.

[22] Wobig H. and Andreeva T. et al., in Proceedings of the 36th EPS Plasma Physics Conference Sofia, Bulgaria (European Conference Abstracts) 2009, Paper P4.192.

[23] Sagara A. et al., Fusion Eng. Des., 81 (2006) 2703.

[24] Sagara A. et al., Nucl. Fusion, 45 (2005) 258. 
[25] Federici G. et al., in Proceedings of the 25th Symposium Fusion Engineering (SOFE), 10-14 June 2013, San Francisco, USA (SOFE) 2013, paper WO1-1.

[26] Knaster J. et al., Nucl. Fusion, 53 (2013) 116001.

[27] Sonato P. et al., in Proceedings of the 3rd International Symposium on Negative Ions, Beams and Sources, (NIBS), Jyväskylä, Finland, AIP Conf. Proc., 1515 (2013) 549.

[28] Romanelli F. et al., in Fusion Electricity: A roadmap to the realization of fusion energy (EFDA, Garching bei München, Germany) 2012, ISBN-13: 978-3000407208.

[29] Hoshino T., Desalination, 359 (2015) 59.

[30] Wang M. et al., Chin. Phys. C, 41 (2017) 030003. 